will need urgent surgery, and $8 \%$ will require non-urgent surgery. The relevant abilities of the Israeli public hospitals were calculated, including the number of emergency department beds, operating rooms, ICU beds, and hospital beds in order to better define the gaps between the needs of the injured patients expected from mega-attacks by terrorists and the abilities of the hospitals in each area of Israel.

Results: Applying these numbers to an urban area in Israel, three potential bottlenecks are possible: (1) inadequate number of ambulances required for the rapid evacuation of the casualties from the site within the first hour; (2) lack of sufficient numbers of intensive care beds in the nearby (close-circle) hospitals; and (3) the lack of a complete picture of the system will not enable optimal coordination of this complex event.

Discussion: Because of the lack of EMS personnel and vehicles at the scene, there will be a need for advanced, prehospital life support and primary triage by experienced paramedics. There should be rapid transport of the moderately and severely injured casualties to the nearby hospitals (close circle), but mild and stress casualties must be delayed at the scene and transported by buses to hospitals outside of the region (second circle). The possible shortage of ICU beds necessitates the early recruitment of both ambulances and helicopters at the hospitals, so they can be ready for secondary evacuation of casualties to second-circle hospitals or to trauma centers. A medical operational center with a good knowledge of the field-level and hospital-level doctrines and a system for data collection and interpretation should assist the coordinator of such an event.

Conclusion: Such an exercise reveals potential bottlenecks in the flow of injured victims from a mega-attack by terrorists and solutions for the problems can be derived from the analysis.

Keywords: ambulances; bottlenecks; casualties; emergency departments; emergency medical services; exercise; helicopters; hospitals; intensive care units; multi-casualty incident; preparedness; surgery; terrorism; treatment

Prehosp Disast Med 2005;20(2):s43-s44

The Strategic National Stockpile Program: Emergency Response Preparedness for Chemical, Biological, and Radiological Disasters through Present and Future Initiatives

N. Pesik

Centers for Disease Control and Prevention, Atlanta, Georgia, USA

The United States Strategic National Stockpile (SNS) Program was established in 1999 as a national resource to deliver medical material to the site of a national emergency. The SNS Program ensures the availability of medicines, antidotes, medical supplies, vaccines, and medical equipment necessary for states and communities to counter the effects of biological pathogens, chemical nerve agents, radiological events, and explosive devices. The SNS Program is designed to deliver medical assets to the site of a national emergency within 12 hours of a federal decision to deploy medical assets. The purpose of this paper is to describe present and future initiatives, which have increased the SNS program's emergency response preparedness for disasters. The SNS Program formulary undergoes continual review and is subject to modifications based on clinical recommendations, improvements in existing pharmaceuticals, or current threat information. A brief overview of formulary modifications will be presented. Recently, the SNS Program also has undertaken a national effort in augmenting state preparedness with forward placement of chemical nerve agent antidotes in cities and states. Through this effort, emergency medical services and hospitals will have access to chemical nerve agent antidotes for immediate use during an event. The SNS Program also continues to address the needs of the pediatric population during disasters. Recent initiatives include modification of 12-hour Push Packages containers, expanding pediatricspecific formulary items, and efforts to reduce medication errors. The SNS Program also has contributed to a review of mechanical ventilation requirements in mass-casualty situations. The basic characteristics of mechanical ventilators for use in mass-casualty situations may help define requirements for programs considering future stockpiling of mechanical ventilators. The SNS program continues to improve its readiness and capabilities to respond to masscasualty events through current and future initiatives.

Keywords: initiatives; response; Strategic National Stockpile (SNS) Prebosp Disast Med 2005;20(2)::44

\section{Predictors of Suicidal Ideation in 1,138 of the 11 September World Trade Center Rescue and Recovery Workers}

\section{R.P. Smith; ${ }^{1}$ G.L. Larkin ${ }^{2}$}

1. Disaster Psychiatry Outreach/Mount Sinai Hospital, New York, New York USA

2. University of Texas Southwestern Medical School, Dallas, Texas USA

A mental health, screening program was initiated together with a medical screening program to assess the physical and mental health of 11,000 workers involved in the World Trade Center rescue and recovery effort in New York City after the terrorist attacks of 11 September 2001. Analyses of predictors of suicidal ideation (SI) are presented on the 1,138 rescue and recovery workers who were evaluated between 16 July-31 December 2002.

Responders completed the following standardized, selfadministered questionnaires: (1) the General Health Questionnaire (GHQ-28); (2) the Post-Traumatic Stress Disorder (PTSD) Symptom Checklist (PCL); (3) the Patient Health Questionnaire (PHQ) modules for Panic Disorder; (4) Generalized Anxiety Disorder; (5) Major Depression; and (6) the CAGE alcohol questionnaire. The questionnaires included five questions about the presence of suicidal ideation.

Results: Of the 1,138 respondents, 1,047 (91\%) were male, 660 (58\%) were white, 125 (11\%) were African-American, and $170(15 \%)$ were Latino. A total of $92(8 \%)$ people endorsed at least one question about SI, and $227(20 \%)$ had post-traumatic stress disorder.

Conclusion: Suicidal ideation was higher in people who met threshold criteria on standardized questionnaires for diagnosis with anxiety disorders. However, surprisingly, hours of exposure to the disaster site, race, alcohol problems, and marital status variables were not associated with increased incidences of SI. 